\title{
Maternal COVID-19 leaves a lasting immunological impression on the fetus
}

\author{
Whether COVID-19 during pregnancy affects the health of children is unclear. Data now show that SARS-CoV-2 \\ infection of mothers can prime the fetal immune response indirectly even when the virus does not infect the fetus.
}

\section{Brahm Coler and Kristina Adams Waldorf}

$\mathrm{N}$ early two years into the COVID-19 pandemic, important questions remain unanswered about the risks that the coronavirus SARS-CoV-2 poses to infants born to mothers who had COVID-19 during pregnancy. There is cause for concern, as activation of the maternal immune system has been shown to adversely affect fetal neurodevelopment and increase the long-term risk of neuropsychiatric disorders in mouse models and in humans ${ }^{1,2}$. In this issue, Gee et al. provide the first evidence linking maternal COVID-19 with dysregulation of the fetal immune system and activation of multiple innate, innate-like and adaptive immune cells in neonatal cord blood ${ }^{3}$. Similar to other published studies showing a very low rate of vertical transmission of SARS-CoV-2, congenital infection in this neonatal cohort may have occurred in only a single case ( 1 of $30(\sim 3 \%))$. What remains unclear is whether and how a maternal SARS-CoV-2 infection affects fetal immunity and development in the absence of fetal viral infection.
Gee et al. demonstrate that neonates born to women with a recent or ongoing maternal SARS-CoV-2 infection at the time of birth - in the absence of a congenital infection - had increased frequencies of neonatal natural killer (NK) cells, $\mathrm{V} \delta 2^{+} \gamma \delta$ $\mathrm{T}$ cells and $\mathrm{FOXP}^{+}$regulatory $\mathrm{T}$ cells, and greater quantities of plasma cytokines ${ }^{3}$

(Fig. 1). Fetal monocyte populations were also perturbed, with increased frequencies of alternative monocytes and decreased frequencies of $\mathrm{CD} 38^{+}$monocytes. Furthermore, regardless of when COVID-19 was diagnosed during pregnancy, a broad increase in a nonspecific, neonatal polyfunctional cytokine response involving populations of $\mathrm{CD} 4^{+} \mathrm{T}$ cells, $\mathrm{CD}^{+}$ T cells, NK cells, NKT cells and $\gamma \delta \mathrm{T}$ cells was identified. A polyfunctional cytokine response refers to the ability of a cell population to produce multiple cytokines at once (such as TNF, IFN- $\gamma$ and IL-17) and represents a marker of cellular activation and maturation, as well as a correlate of protective immunity when specifically targeted to a virus ${ }^{4}$. The increased $\mathrm{T}$ cell cytokine functionality noted by Gee et al. was evaluated in a subset of neonates and was found not to be specific for SARS-CoV-2 peptides in seven of eight neonates $(88 \%)^{3}$. These data indicate that fetal immune imprinting due to a maternal SARS-CoV-2 infection during pregnancy is probably not a result of direct exposure to the virus and is instead a result of fetal exposure to an inflammatory environment.

A striking finding by Gee et al. was that some changes in fetal immunity associated with maternal COVID-19 were time dependent ${ }^{3}$. Perturbations in the frequency of neonatal NK cells and $\mathrm{V} \delta 2^{+} \gamma \delta$

$\mathrm{T}$ cells seemed to recover with a longer interval between maternal COVID-19 and birth. Interestingly, there was an inverse correlation between a higher frequency of cord blood NK cells and time (days) since COVID-19 diagnosis. Furthermore, the concentration of the chemokine CXCL8 in cord plasma correlated with activation of NK cells and the percentage of NKT cells detected. Notably, the polyfunctional cytokine response in multiple immune cell

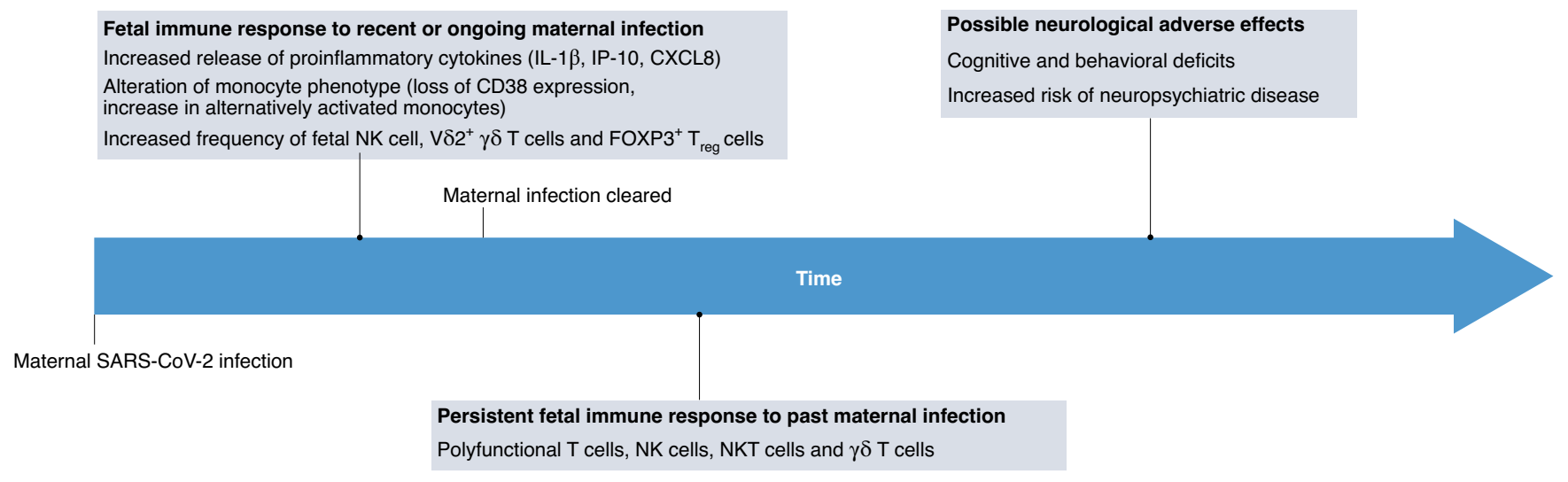

Fig. 1 | Timeline of possible effects of maternal SARS-CoV-2 infection on fetal immunity. During the early stages of maternal SARS-CoV-2 infection, the frequency of immune cells and production of cytokines is altered in the fetus. Later, after the maternal infection is cleared, signs of alteration of the fetal immune system persist, with evidence of polyfunctional immune cells. The concern is that these inflammatory changes might result in subsequent fetal neurological impairment. Although there is no evidence at present to indicate this is the case for SARS-CoV-2, such neurological adverse effects have been recorded in other contexts. 
populations was maintained even when maternal COVID-19 occurred many months before delivery (time interval between diagnosis and delivery in the group that had recovered from COVID-19: median, 48.5 days; range, $22-221$ days). Similar to these findings, a change in the maturation and activation status of multiple fetal immune effectors was described previously in the setting of human congenital Chagas disease (due to infection with Trypanosoma cruzi) and in fetal rhesus monkeys exposed to IL- $1 \beta$ via inoculation into the amniotic fluid $^{5,6}$. Together these data indicate that fetal exposure to a maternal SARS-CoV-2 infection induces a defined and time-dependent fetal immune response even in the absence of congenital infection.

Although the development of polyfunctional $\mathrm{T}$ cells and innate-like cells during fetal life might confer protective benefits against viral threats such as SARS-CoV-2, these immune responses probably come at a cost. Fetal development functions as a multi-faceted and tightly regulated symphony with the objective of developing all bodily organs in a short window of time. Activation of immune effector cells and induction of inflammatory cytokines threatens the fine balance needed to support developmental programming, particularly of the fetal brain. Changes in immune effector cell activation and proinflammatory cytokine production can negatively affect fetal neurodevelopment. Alarmingly, Gee et al. found that fetal cytokine levels were, in some cases, as high as those in the mother actively or recently infected with SARS-CoV- $2^{3}$. In the absence of a control group of fetuses not exposed to SARS-CoV-2, the authors compared the umbilical cord plasma cytokines of infants born to women with a recent or ongoing SARS-CoV-2 infection with those of infants from women who had recovered from SARS-CoV-2 infection. Although the difference between these groups in cytokine distribution in cord blood was statistically significant only for IL-10 (higher in the group with recent or ongoing infection), the absolute level of several cytokines (such as IL-1 $\beta$, IP-10 and CXCL8) was elevated in $~ 25-50 \%$ of neonates in the group with recent or ongoing maternal SARS-CoV-2 infection. As an apex inflammatory cytokine, IL- $1 \beta$ is a marker of neonatal organ injury that can induce arrest of organogenesis of the fetal lungs, intestine and brain, as well as induce extensive neuronal injury ${ }^{7}$. The changes in developmental programming induced by elevations in fetal cytokines are probably subtle, difficult to detect clinically and evident only over time.
Neonatal T cells and other immune cells should remain somewhat hypofunctional at birth and poised toward a tolerogenic profile, for important biological reasons. A few days after birth, a wave of bacteria colonizes the neonatal intestine in a process crucial to the establishment of a healthy intestinal microbiota. An immune repertoire composed of hypofunctional $\mathrm{T}$ cells and other effector cells is probably advantageous during this important event to prevent an immune response that injures the neonatal intestine. A greater capacity of neonatal effector cells to mount a functional immune response might increase the risk of a catastrophic intestinal inflammatory response.

Interestingly, a coronavirus epidemic in France in 1979-1980 was associated with a spike in cases of neonatal necrotizing enterocolitis ${ }^{8}$, a life-threatening inflammation of the gastrointestinal tract that is typically a rare complication occurring only in low-birthweight preterm infants. In intestinal tissues from ten infants with necrotizing enterocolitis in this cohort, coronavirus-like particles were identified (by electron microscopy) in the damaged intestinal epithelial cells, which suggests that direct infection of the neonatal intestine by a coronavirus is possible ${ }^{9}$. Maternal COVID-19 has also been associated with unexpected cases of necrotizing enterocolitis in term and late-preterm infants ${ }^{10,11}$. Although necrotizing enterocolitis seems to be rare in response to maternal SARS-CoV-2 infection, whether fetal exposure to new SARS-CoV-2 variants might induce greater neonatal disease and intestinal inflammation similar to exposure to other coronaviruses is unclear.

The findings presented by Gee et al. ${ }^{3}$ warrant a thorough, longitudinal study of potential adverse events conferred by SARS-CoV-2 on the developing fetus that might not be apparent for many years. Activation of the maternal immune system caused by exposure to pathogens or inflammation during critical periods of fetal neurodevelopment is a risk factor for behavioral deficits and psychiatric illness later in childhood and adulthood ${ }^{1,12}$. A striking association between maternal viral disease, abnormal fetal brain development and a higher lifetime risk of neuropsychiatric disorders has been identified across a wide variety of prenatal infections ${ }^{1,2}$. Our prior research linking a spectrum of maternal infections with an increased lifetime risk for neuropsychiatric disorder in children demonstrated that a large study population followed for at least 5 years is needed to identify differences in rates of autism spectrum disorder ${ }^{1}$. Rodent models can demonstrate the link between prenatal infection and neurological disorders in a much shorter time period, because viral mimetics administered during pregnancy can recapitulate a spectrum of behavioral abnormalities in the offspring. However, long-term studies of children exposed to SARS-CoV-2 in utero are needed to identify whether the lifetime risk for neuropsychiatric disorders is elevated.

In summary, these new data show that fetal exposure to SARS-CoV-2 acutely increases the frequencies of fetal NK cell and $\gamma \delta$ T cell immune effector cells, and also accelerates the maturation and non-specific activation of multiple fetal immune cell populations. Although the effect of these changes on neonatal immunity might not be known for many years, fetal exposure to a maternal SARS-CoV-2 infection - even in the absence of a congenital infection - can imprint the fetal immune system. Better understanding is needed of the role of the placenta in protecting the fetus from SARS-CoV-2 congenital infection while simultaneously permitting immune crosstalk that might fuel activation of the fetal immune system and alter developmental programs. Although recent developments have provided a great deal of information about SARS-CoV-2, the effect on the developing fetus is only beginning to be understood.

\section{Brahm Coler ${ }^{1,2}$ and \\ Kristina Adams Waldorf (D) 1,3凶 \\ ${ }^{1}$ Department of Obstetrics \& Gynecology, University of Washington, Seattle, WA, USA. ${ }^{2}$ Elson S. Floyd College of Medicine, Washington State University, Spokane, WA, USA. ${ }^{3}$ Department of Global Health, University of Washington, Seattle, WA, USA. \\ $凶_{e-m a i l: a d a m s k @ u w . e d u}$}

Published online: 15 November 2021 https://doi.org/10.1038/s41590-021-01072-3

References

1. Al-Haddad, B. J. S. et al. JAMA Psychiatry 76, 594-602 (2019).

2. Al-Haddad, B. J. S. et al. Am. J. Obstet. Gynecol. 221, 549-562 (2019).

3. Gee, S. et al. Nat. Immunol. https://doi.org/10.1038/s41590-02101049-2 (2021).

4. Harari, A. et al. Immunol. Rev. 211, 236-254 (2006)

5. Dauby, N. et al. PLoS Negl. Trop. Dis. 3, e571 (2009).

6. Kallapur, S. G. et al. J. Immunol. 191, 1102-1109 (2013).

7. Nadeau-Vallee, M. et al. J. Immunol. 198, 2047-2062 (2017).

8. Chany, C., Moscovici, O., Lebon, P. \& Rousset, S. Pediatrics 69 209-214 (1982).

9. Rousset, S. et al. Pediatrics 73, 218-224 (1984).

10. Wu, Y. T. et al. PLoS Med. 17, el003195 (2020).

11. Liu, P. et al. J. Allergy Clin. Immunol. 146, 101-109.e101 (2020).

12. Boulanger-Bertolus, J., Pancaro, C. \& Mashour, G. A. Front. Behav. Neurosci. 12, 230 (2018).

The authors declare no competing interests. 